\section{Further evaluation of Vigabatrin therapy in 4-hydroxybutyric aciduria}

\author{
C.Jakobs ${ }^{1}$, T. Michael ${ }^{2}$, E.Jaeger ${ }^{2}$, \\ J. Jaeken ${ }^{3}$, and K.M. Gibson ${ }^{4}$ \\ ${ }^{1}$ Department of Paediatrics, Free University Hospital, Amsterdam, \\ The Netherlands \\ ${ }^{2}$ University Children's Hospital, Free University, Berlin, \\ Federal Republic of Germany \\ ${ }^{3}$ Department of Paediatrics, University Hospital Gasthuysberg, Leuven, \\ Belgium \\ ${ }^{4}$ Metabolic Disease Center, Baylor University Medical Center, \\ Dallas, Texas, USA
}

Sir: Succinic semialdehyde dehydrogenase (SSADH) deficiency is an inborn error of gamma-aminobutyric acid (GABA) metabolism [3] accompanied by urinary excretion of 4-hydroxybutyric (4-HB) acid, a compound with neuropharmacological properties [5]. Patients present with nonprogressive ataxia, hypotonia, psychomotor retardation, hyperactivity, autistic features and/or convulsions [6]. Jaeken and coworkers [2] reported a successful therapeutic trial with the anticonvulsant Vigabatrin in one patient. In a subsequent report by Gibson et al. [1] Vigabatrin therapy was without clinical improvement in another patient. We wish to report our results of Vigabatrin therapy in SSADH deficiency.

Our patient was the second child of consanguineous Turkish parents. She showed gross retardation of motor and psychosocial development, hypotonia and ataxia. Hypotonic cerebral palsy was diagnosed. She sat unsupported at 12 months and walked at 27 months. Gait remained broad based and required support. She did not speak nor comprehend speech, could not feed or dress herself and had no control of bladder and bowel. There was little functional use of the hands. Moreover there were autistic features and severe physical restlessness with insomnia. She indulged in repetitive motor activities, wandering aimlessly for hours during the day and night. She required constant supervision, occasionally with restraint. She developed disruptive and aggressive behaviour with explosive outbursts. At 4 years of age grand mal and myoclonic seizure activity were noted, partially controlled by sodium valproate. At 8 years of age atypical absence seizures developed and ethosuccimide therapy was added. However, seizures could not be completely controlled. At 8 years old SSADH deficiency was diagnosed [4, patient 2]. At 12 years of age Vigabatrin therapy was started with increasing doses from 500 to $3500 \mathrm{mg} / \mathrm{d}$. Within 2 weeks of this therapy there was neurological improvement. There was noticeable improvement in locomotion. Her gait became non-atactic and support was no longer required. Sodium valproate and ethosuccimide therapy was discontinued as seizures resolved. Over the next several weeks there was a modest improvement in mental development. Her IQ improved slightly from $<20$ to $20-50$. She began to develop speech consisting of about one dozen intelligible words. She fed herself, picked up a cup, drank and managed a spoon, dressed and undressed herself with assistance and showed bladder control during the day. She could execute simple orders and began to imitate domestic duties. There was slight improvement in au-

Abbreviations: $\mathrm{GABA}=$ gamma-aminobutyric acid; 4 -HB $=4$-hydroxybutyric acid; SSADH = succinic semialdehyde dehydrogenase tistic behaviour and she began to display emotions. However, as she was undergoing behavioural therapy simultaneously with Vigabatrin treatment, it is difficult to evaluate which therapeutic regimen was responsible for improved mental development. There was no improvement in hyperactivity, insomnia or agressive behaviour. Agressiveness may have been the result of institutional restraint for hyperactivity and may or may not be a manifestation of her inborn error of metabolism.

Concentrations of 4-HB acid in physiological fluids showed a tendency to decrease during Vigabatrin therapy. Plasma 4HB acid levels dropped to $66 \%$ of pretreatment levels after 2 weeks and to $45 \%$ after 9 months. Also levels in CSF decreased with concomitant increase of CSF GABA levels (before Vigabatrin $189 \mathrm{nmol} / 1$ : during $460 \mathrm{nmol} / 1$ ).

The patient described by Jaeken and coworkers [2] showed a persistent clinical improvement during Vigabatrin therapy. CSF 4-HB acid levels fell $70 \%$ from pretreatment levels, while free $\mathrm{GABA}$ concentrations showed an almost sixfold increase. However, in the patient described by Gibson and coworkers [1] there was no significant change in the CSF 4-HB acid levels and no clinical improvement. It should be mentioned that this patient showed a complete enzymatic block, while in most other patients there is some residual enzyme activity. We feel that early institution of Vigabatrin therapy will lead to an improved prognosis for both neurological parameters and mental development.

\section{References}

1. Gibson KM, De Vivo DC, Jakobs C (1989) Vigabatrin therapy in a patient with succinic semialdehyde dehydrogenase deficiency. Lancet II: $1105-1106$

2. Jaeken J, Casaer P, DeCock P, Francois B (1989) Vigabatrin in GABA metabolism disorders. Lancet I: 1074

3. Jakobs C, Kneer J, Rating D, Hanefeld F, Divry P, Hermier M (1984) 4Hydroxybutyric aciduria: a new inborn error of metabolism. II. Biochemical findings. J Inherited Metab Dis 7 [Suppl] 1:92-94

4. Jakobs C, Smit LME, Kneer J, Michael T, Gibson KM (1990) The first adult case with 4-hydroxybutyric aciduria. J Inherited Metab Dis 13: 341-344

5. Mamelak M (1989) Gammahydroxybutyrate: an endogenous regulator of energy metabolism. Neurosci Biobehav Rev 13:187-198

6. Rating D, Hanefeld F, Siemens H, Kneer $J$, Jakobs C, Hermier M, Divry P (1984) 4-Hydroxybutyric aciduria: a new inborn error of metabolism: I. Clinical review. J Inherited Metab Dis 7 [Suppl] 1:90-92

\section{Severe caffeine intoxication in a preterm neonate}

\section{J.N. van den Anker, H.T. M. Jongejan, and P. J. J. Sauer}

Department of Paediatrics, Erasmus University and University Hospital Rotterdam/Sophia Children's Hospital, The Netherlands

Received January 20, 1992 / Accepted January 20, 1992

Sir: Episodes of prolonged apnoea accompanied by bradycardia occur frequently in preterm infants and may lead to recurrent hypoxaemia and neurological damage. Since 1973 methylxanthines have undergone clinical trials for the treatment of apnoea of undetermined origin and have shown satisfactory responses [4]. 
Our policy is to treat apnoea of prematurity with caffeine. A loading dose of $10 \mathrm{mg} / \mathrm{kg}$ is followed by a maintenance dose of $2.5 \mathrm{mg} / \mathrm{kg}$ once daily to achieve desired serum concentrations $(5-20 \mathrm{mg} / \mathrm{l})$. Preterm infants may be relatively resistant to poisoning by theophylline and caffeine: concentrations of up to $80 \mathrm{mg} / 1$ (theophylline) have resulted in sustained tachycardia as the only sign of toxicity $[1,2]$.

We here describe severe caffeine intoxication in a preterm neonate. A Caucasian male was delivered by caesarean section (gestational age: 33.7 weeks; weight $1625 \mathrm{~g}$ ) and showed signs of respiratory insufficiency necessitating artificial ventilation for 5 days; otherwise his clinical course was uneventful. Apnoea of prematurity was treated with caffeine. On the 7th day after birth he was transferred to another hospital. There were no changes in therapy but 2 days after admission the child deteriorated. Tachypnoea, tachycardia, compromised circulation, vomiting and convulsions were the presenting symptoms. Septicaemia was suspected and after blood, urine and CSF cultures were taken, broad spectrum antibiotics were started. An attempt to treat the tachycardia with digoxin was unsuccessful. There was a search for metabolic disorders because clinical deterioration started after the introduction of enteral feedings. Analysis of the urine showed a tremendous amount of caffeine and the serum caffeine concentration was $346 \mathrm{mg} / \mathrm{l}$. Caffeine was stopped and there was a rapid improvement in clinical condition together with a decrease in serum caffeine concentration. After 9 days the serum caffeine concentration was 32.9 $\mathrm{mg} / \mathrm{l}$. He is now 18 months old and his psychomotor development is excellent. A thorough investigation revealed an error during the processing of the caffeine in the pharmacy. The parents, management of the hospital and government were informed.

Ehlers et al. [3] described a patient with a serum theophylline concentration of $190 \mathrm{mg} / \mathrm{l}$ who survived albeit with permanent neurological deficits.

As far as we are aware this is the highest serum caffeine concentration ever reported in a preterm infant.

This case report of an extreme caffeine intoxication shows no long-term side-effects at the age of 18 months and clearly demonstrates that we should always consider iatrogenic causes of sudden deterioration in children, especially when they receive drugs.

\section{References}

1. Aranda JV, Gorman W, Bergsteinsson H, et al (1977) Efficacy of caffeine in treatment of apnoea in the low-birth-weight-infant. J Pediatr $90: 467$

2. Cole GF, Davies DP (1980) Theophylline poisoning. BMJ 280:52

3. Ehlers SM, Zaske DE, Sawchuk RJ (1978) Massive theophylline overdose: rapid elimination by charcoal hemoperfusion. JAMA $240: 474-475$

4. Kuzemko JA, Paala J (1973) Apnoeic attacks in the newborn treated with aminophylline. Arch Dis Child 48:404-406

\section{VACTERL plus hydrocephalus: a monogenic lethal condition}

\section{J.Kunze, S. Huber-Schumacher, and M. Vogel}

Kinderklinik der Freien Universität, Heubnerweg 6, W-1000 Berlin 19, Federal Republic of Germany

Received January 20, 1992 / Accepted January 20, 1992

Sir: The acronym VACTERL describes a non-random association of vertebral anomalies, anal atresia, cardio-vascular anomalies, tracheo-oesophageal fistula with renal dysplasia and limb

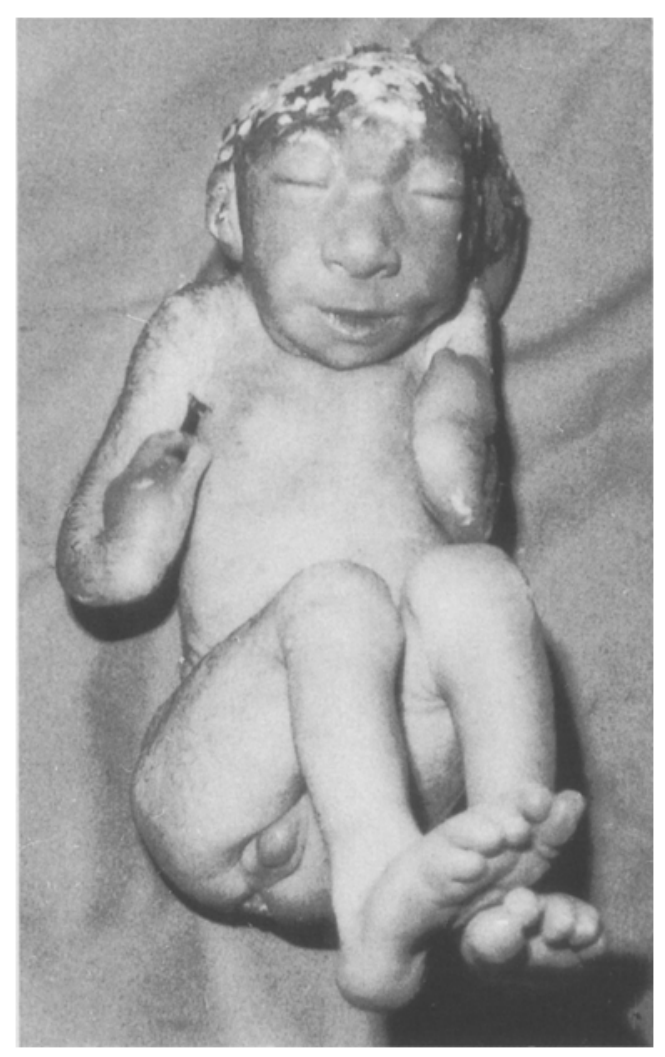

Fig. 1. Twin 1: male with hypertelorism, symmetric exophthalmia, dysplastic helices, macrostomia, macroglossia, microcephalia, marked occipital meningo-encephalocele, multiple cerebral malformations (malformed brain stem, absence of olives, pons and cerebelium), hypoplastic adrenals, atresia ani, bilateral absence of radii and thumbs, shortened ulna with marked bowing on both sides, long fingers, single umbilical artery, hypoplasia of the arteria iliaca dextra, atrial septal defect, oesophageal atresia with tracheo-oesophageal fistula, duodenal atresia, multiple spleens, mesenterium ileocolicum commune, short bowel and horseshoe kidneys

defects. Recent reports suggest that the VACTERL association can be caused by a single mutation [1]. In addition to classical VATER-type defects these patients have central nervous system abnormalities including hydrocephalus. Their prognosis is poor; in one report all patients died during the neonatal period [1].

We observed three male patients born to a healthy 19/20year-old Arabian woman and her 26/27-year-old husband who is her first degree cousin.

Twin 1 had low-set malformed ears, mandibular micrognathia, absence of cerebellum, hypoplastic adrenals, anal atresia, bilateral absence of radius and thumbs, short ulna on the left side, bowed ulna on the right side, cutaneous syndactyly between digits II/III on the left hand, right-sided club foot, hypospadias, hypoplastic lungs, incomplete lung lobation, multiple accessory spleens, mesenterium ileocolicum commune, and horseshoe kidneys. Abnormalities of twin 2 and patient 3 are shown in Fig. 1 and 2.

Iafolla et al. [2] in their report on three patients with VACTERL association and hydrocephalus suggest that the disorder is distinct from sporadic VACTERL association. Etiologically, we agree but pathogenetically they appear to be closely related. It has been hypothesized that associations are derivatives of nonspecific disruptive events acting on multiple developmental fields [3]. Now the observation of a single mutation causing the VACTERL association and hydrocephaly imparts that multiple developmental field defects can result from a genetic cause, i.e. are primary rather than secondary 